\title{
On the Security of TANDEM-DM
}

\author{
Ewan Fleischmann, Michael Gorski, and Stefan Lucks
}

Bauhaus-University Weimar, Germany

\{ewan.fleischmann, michael.gorski, stefan.lucks\}@uni-weimar.de

\begin{abstract}
We provide the first proof of security for TANDEM-DM, one of the oldest and most well-known constructions for turning a block cipher with $n$-bit block length and $2 n$-bit key length into a $2 n$-bit cryptographic hash function. We prove, that when TANDEM-DM is instantiated with AES-256, block length 128 bits and key length 256 bits, any adversary that asks less than $2^{120.4}$ queries cannot find a collision with success probability greater than $1 / 2$. We also prove a bound for preimage resistance of TANDEM-DM.

Interestingly, as there is only one practical construction known turning such an $(n, 2 n)$ bit block cipher into a $2 n$-bit compression function that has provably birthday-type collision resistance (FSE'06, Hirose), TANDEM-DM is one out of two constructions that has this desirable feature.
\end{abstract}

Keywords: Cryptographic hash function, block cipher based, proof of security, double-block length, ideal cipher model, Tandem-DM.

\section{Introduction}

A cryptographic hash function is a function which maps an input of arbitrary length to an output of fixed length. It should satisfy at least collision-, preimageand second-preimage resistance and is one of the most important primitives in cryptography 23].

Block Cipher-Based Hash Functions. Since their initial design by Rivest, MD4family hash functions (e.g. MD4, MD5, RIPEMD, SHA-1, SHA2 [26, 27, 29, 30]) have dominated cryptographic practice. But in recent years, a sequence of attacks on these type of functions [7, 10, 37, 38] has led to a generalized sense of concern about the MD4-approach. The most natural place to look for an alternative is in block cipher-based constructions, which in fact predate the MD4-approach 22. Another reason for the resurgence of interest in block cipher-based hash functions is due to the rise of size restricted devices such as RFID tags or smart cards: A hardware designer has to implement only a block cipher in order to obtain an encryption function as well as a hash function. But since the output length of most practical encryption functions is far too short for a collision resistant hash function, e.g. 128-bit for AES, one is mainly interested in sound design principles for double block length (DBL) hash functions [2]. A DBL hash-function uses a block cipher with $n$-bit output as the building block by which it maps possibly long strings to $2 n$-bit ones.

O. Dunkelman (Ed.): FSE 2009, LNCS 5665, pp. 84-103, 2009.

(C) International Association for Cryptologic Research 2009 
Our Contribution. Four 'classical' DBL hash functions are known: MDC-2, MDC-4, Abreast-DM and TAnDEm-DM 3, 4, 20]. At EUROCRYPT'07, Steinberger [35] proved the first security bound for the hash function MDC2: assuming a hash output length of 256 bits, any adversary asking less than $2^{74.9}$ queries cannot find a collision with probability greater than $1 / 2$.

In this paper, we prove the first security bound for the compression function TANDEM-DM in terms of collision resistance and preimage resistance. We will give an upper bound for success if an adversary is trying to find a collision. By assuming a hash output length of 256 bits, any adversary asking less than $2^{120.4}$ queries cannot find a collision with probability greater than $1 / 2$. We will also prove an upper bound for success if an adversary is trying to find a preimage. This bound is rather weak as it essentially only states, that the success probability of an adversary asking strictly less than $2^{n}$ queries is asymptotically negligible.

Beyond providing such a proof of security for TANDEM-DM in the first place, our result even delivers one of the most secure rate $1 / 2 \mathrm{DBL}$ compression functions known. The first practical DBL compression function with rate $1 / 2$ (without bitfixing and other artificial procedures like employing two different block ciphers) that has a birthday-type security guarantee was presented at FSE'06 by Hirose [13. He essentially states (see Appendix B for more details) that no adversary asking less than $2^{124.55}$ queries, again for $2 n=256$, can find a collision with probability greater then 1/2. These two compression functions (Hirose's FSE '06 proposal and TANDEM-DM) are the only rate $1 / 2$ practical compression functions that are known to have a birthday-type security guarantee.

Outline. The paper is organized as follows: Section 2 includes formal notations and definitions as well as a review of related work. In Section 3 we proof that an adversary asking less than $2^{120.4}$ oracle queries has negligible advantage in finding a collision for the TANDEM-DM compression function. A bound for preimage resistance of TANDEM-DM is given in Section 4. In Section 5 we discuss our results and conclude the paper.

\section{Preliminaries}

\subsection{Iterated DBL Hash Function Based on Block Ciphers}

Ideal Cipher Model. An $(n, k)$-bit block cipher is a keyed family of permutations consisting of two paired algorithms $E:\{0,1\}^{n} \times\{0,1\}^{k} \rightarrow\{0,1\}^{n}$ and $E^{-1}$ : $\{0,1\}^{n} \times\{0,1\}^{k} \rightarrow\{0,1\}^{n}$ both accepting a key of size $k$ bits and an input block of size $n$ bits. For simplicity, we will call it an $(n, k)$-block cipher. Let $\mathrm{BC}(n, k)$ be the set of all $(n, k)$-block ciphers. Now, for any one fixed key $K \in\{0,1\}^{k}$, decryption $E_{K}^{-1}=E^{-1}(\cdot, K)$ is the inverse function of encryption $E_{K}=E(\cdot, K)$, so that $E_{K}^{-1}\left(E_{K}(x)\right)=x$ holds for any input $X \in\{0,1\}^{n}$.

The security of block cipher based hash functions is usually analyzed in the ideal cipher model [2, 9, 17]. In this model, the underlying primitive, the block cipher $E$, is modeled as a family of random permutations $\left\{E_{k}\right\}$ whereas the random permutations are chosen independently for each key $K$, i.e. formally $E$ is selected randomly from $\mathrm{BC}(n, k)$. 
DBL Compression Functions. Iterated DBL hash functions with two block cipher calls in their compression function are discussed in this article. A hash function $H:\{0,1\}^{*} \rightarrow\{0,1\}^{2 n}$ can be built by iterating a compression function $F:\{0,1\}^{3 n} \rightarrow\{0,1\}^{2 n}$ as follows: Split the padded message $M$ into $n$-bit blocks $M_{1}, \ldots, M_{l}$, fix $\left(G_{0}, H_{0}\right)$, apply $\left(G_{i}, H_{i}\right)=F\left(G_{i-1}, H_{i-1}, M_{i}\right)$ for $i=1, \ldots, l$ and finally set $H(M):=\left(G_{l}, H_{l}\right)$. Let the compression function $F$ be such that

$$
\left(G_{i}, H_{i}\right)=F\left(G_{i-1}, H_{i-1}, M_{i}\right)
$$

where $G_{i-1}, H_{i-1}, G_{i}, H_{i}, M_{i} \in\{0,1\}^{n}$. We assume that the compression function $F$ consists of $F_{T}$, the top row, and $F_{B}$, the bottom row. We explicitly allow the results of $F_{T}$ to be fed into the calculation of $F_{B}$. Each of the component functions $F_{B}$ and $F_{T}$ performs exactly one call to the block cipher and can be defined as follows:

$$
\begin{aligned}
& G_{i}=F_{T}\left(G_{i-1}, H_{i-1}, M_{i}\right)=E\left(X_{T}, K_{T}\right) \oplus Z_{T}, \\
& H_{i}=F_{B}\left(G_{i}, G_{i-1}, H_{i-1}, M_{i}\right)=E\left(X_{B}, K_{B}\right) \oplus Z_{B},
\end{aligned}
$$

where $X_{T}, K_{T}, Z_{T}$ are uniquely determined by $G_{i-1}, H_{i-1}, M_{i}$ and $X_{B}, K_{B}, Z_{B}$ are uniquely determined by $G_{i}, G_{i-1}, H_{i-1}, M_{i}$.

We define the rate $r$ of a block cipher based compression/hash function $F$ by

$$
r=\frac{\left|M_{i}\right|}{(\text { number of block cipher calls in } \mathrm{F}) \times n}
$$

It is a measure of efficiency for such block cipher based constructions.

\subsection{The Tandem-DM Compression Function}

The TANDEM-DM compression function was proposed by Lai and Massey at EUROCRYPT'92 [20]. It uses two cascaded Davies-Meyer [2] schemes. The compression function is illustrated in Figure 1 and is formally given in Definition 1

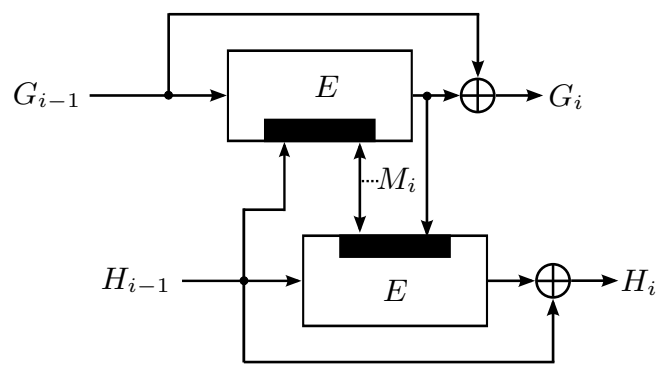

Fig. 1. The compression function TANDEM-DM $F^{T D M}$ where $E$ is an $(n, 2 n)$ block cipher, the small rectangle inside the cipher rectangle indicates which input is used as key 
Definition 1. Let $F^{T D M}:\{0,1\}^{2 n} \times\{0,1\}^{n} \rightarrow\{0,1\}^{2 n}$ be a compression function such that $\left(G_{i}, H_{i}\right)=F^{T D M}\left(G_{i-1}, H_{i-1}, M_{i}\right)$ where $G_{i}, H_{i}, M_{i} \in\{0,1\}^{n}$. $F^{T D M}$ is built upon an $(n, 2 n)$ block cipher $E$ as follows:

$$
\begin{aligned}
W_{i} & =E\left(G_{i-1}, H_{i-1} \mid M_{i}\right) \\
G_{i} & =\mathrm{F}_{T}\left(G_{i-1}, H_{i-1}, M_{i}\right)=W_{i} \oplus G_{i-1} \\
H_{i} & =\mathrm{F}_{B}\left(G_{i-1}, H_{i-1}, M_{i}\right)=E\left(H_{i-1}, M_{i} \mid W_{i}\right) \oplus H_{i-1} .
\end{aligned}
$$

\subsection{Related Work}

Our work is largely motivated by Steinberger [35] in order to provide rigorous proofs for well-known block cipher based hash functions. As is reviewed in the following, there are many papers on hash functions composed of block ciphers.

Schemes with non-optimal or unknown collision resistance. The security of SBL hash functions against several generic attacks is discussed by Preneel et al. in [28]. They concluded that 12 out of 64 hash functions are secure against the attacks. However, formal proofs were first given by Black et al. 2] about 10 years later. Their most important result is that 20 hash functions - including the 12 mentioned above - are optimally collision resistant. Knudsen et al. [18] discussed the insecurity of DBL hash functions with rate 1 composed of $(n, n)$-block ciphers. Hohl et al. 14] analyzed the security of DBL compression functions with rate 1 and 1/2. Satoh et al. [33] and Hattoris et al. [11] discussed DBL hash functions with rate 1 composed of $(n, 2 n)$ block ciphers. MDC-2 and MDC-4 [15, 1, 4] are $(n, n)$-block cipher based DBL hash functions with rates $1 / 2$ and $1 / 4$, respectively. Steinberger [35] proved that for MDC-2 instantiated with, e.g., AES-128 no adversary asking less than $2^{74.9}$ can find a collision with probability greater than $1 / 2$. Nandi et al. [25] proposed a construction with rate $2 / 3$ but it is not optimally collision resistant. Furthermore, Knudsen and Muller [19] presented some attacks against it. At EUROCRYPT'08 and CRYPTO'08, Steinberger [31, 32] proved some security bounds for fixed-key $(n, n)$-block cipher based hash functions, i.e. permutation based hash functions, that all have small rates and low security guarantees. None of these schemes/techniques mentioned so far are known to have birthday-type collision resistance.

Schemes with Birthday-Type Collision Resistance. Merkle 24] presented three DBL hash functions composed of DES with rates of at most 0.276. They are optimally collision resistant in the ideal cipher model. Lucks 21] gave a rate 1 DBL construction with birthday-type collision resistance using a $(n, 2 n)$ block cipher, but it involves some multiplications over $\mathbb{F}_{128}$. Hirose [12] presented a class of DBL hash functions with rate $1 / 2$ which are composed of two different and independent $(n, 2 n)$ block ciphers that have birthday-type collision resistance. At FSE'06, Hirose [13] presented a rate $1 / 2$ and $(n, 2 n)$ block cipher based DBL hash function that has birthday-type collision resistance. As he stated the proof only for the hash function, we have given the proof for his compression function in Appendix B. 


\section{Collision Resistance}

In this section we will discuss the collision resistance of the compression function TANDEM-DM.

\subsection{Defining Security - Collision Resistance of a Compression Function (Pseudo Collisions)}

Insecurity is quantified by the success probability of an optimal resource-bounded adversary. The resource is the number of backward and forward queries to an ideal cipher oracle $E$. For a set $S$, let $z \stackrel{R}{\leftarrow} S$ represent random sampling from $S$ under the uniform distribution. For a probabilistic algorithm $\mathcal{M}$, let $z \stackrel{R}{\leftarrow} \mathcal{M}$ mean that $z$ is an output of $\mathcal{M}$ and its distribution is based on the random choices of $\mathcal{M}$.

An adversary is a computationally unbounded but always-halting collisionfinding algorithm $\mathcal{A}$ with access to an oracle $E \in \mathrm{BC}(n, k)$. We can assume (by standard arguments) that $\mathcal{A}$ is deterministic. The adversary may make a forward query $(K, X)_{f w d}$ to discover the corresponding value $Y=E_{K}(X)$, or the adversary may make a backward query $(K, Y)_{b w d}$, so as to learn the corresponding value $X=E_{K}^{-1}(Y)$ for which $E_{K}(X)=Y$. Either way the result of the query is stored in a triple $\left(X_{i}, K_{i}, Y_{i}\right)$ and the query history, denoted $\mathcal{Q}$, is the tuple $\left(Q_{1}, \ldots, Q_{q}\right)$ where $Q_{i}=\left(X_{i}, K_{i}, Y_{i}\right)$ is the result of the $i$-th query and $q$ is the total number of queries made by the adversary. The value $X_{i} \oplus Y_{i}$ is called 'XOR'-output of the query. Without loss of generality, it is assumed that $\mathcal{A}$ asks at most only once on a triplet of a key $K_{i}$, a plaintext $X_{i}$ and a ciphertext $Y_{i}$ obtained by a query and the corresponding reply.

The goal of the adversary is to output two different triplets, $(G, H, M)$ and $\left(G^{\prime}, H^{\prime}, M^{\prime}\right)$, such that $F(G, H, M)=F\left(G^{\prime}, H^{\prime}, M^{\prime}\right)$. We impose the reasonable condition that the adversary must have made all queries necessary to compute $F(G, H, M)$ and $F\left(G^{\prime}, H^{\prime}, M^{\prime}\right)$. We will in fact dispense the adversary from having to output these two triplets, and simply determine whether the adversary has been successful or not by examining its query history $\mathcal{Q}$. Formally, we say that $\operatorname{Colv}(\mathcal{Q})$ holds if there is such a collision and $\mathcal{Q}$ contains all the queries necessary to compute it.

Definition 2. (Collision resistance of a compression function) Let $F$ be a block cipher based compression function, $F:\{0,1\}^{3 n} \rightarrow\{0,1\}^{2 n}$. Fix an adversary $\mathcal{A}$. Then the advantage of $\mathcal{A}$ in finding collisions in $F$ is the real number

$$
\begin{gathered}
\operatorname{Adv}_{F}^{\operatorname{CoLL}}(\mathcal{A})=\operatorname{Pr}\left[E \stackrel{R}{\leftarrow} \mathrm{BC}(n, k) ;\left((G, H, M),\left(G^{\prime}, H^{\prime}, M^{\prime}\right)\right) \stackrel{R}{\leftarrow} \mathcal{A}^{E, E^{-1}}:\right. \\
\left.\left((G, H, M) \neq\left(G^{\prime}, H^{\prime}, M^{\prime}\right)\right) \wedge F(G, H, M)=F\left(G^{\prime}, H^{\prime}, M^{\prime}\right)\right] .
\end{gathered}
$$

For $q \geq 1$ we write

$$
\operatorname{Adv}_{F}^{\mathrm{CoLL}}(q)=\max _{\mathcal{A}}\left\{\mathbf{A d v}_{F}^{\mathrm{CoLL}}(\mathcal{A})\right\}
$$

where the maximum is taken over all adversaries that ask at most $q$ oracle queries, i.e. $E$ and $E^{-1}$ queries. 


\subsection{Security Results}

Our discussion will result in a proof for the following upper bound:

Theorem 1. Let $F:=F^{T D M}$ be as in Definition 1 and $n, q$ be natural numbers with $q<2^{n}$. Let $N^{\prime}=2^{n}-q$ and let $\alpha$ be any positive number with eq/ $N^{\prime} \leq \alpha$ and $\tau=\alpha N^{\prime} / q$ (and $e^{x}$ being the exponential function). Then

$$
\operatorname{Adv}_{F}^{\mathrm{COLL}}(q) \leq q 2^{n} e^{q \tau(1-\ln \tau) / N^{\prime}}+4 q \alpha / N^{\prime}+6 q /\left(N^{\prime}\right)^{2}+2 q /\left(N^{\prime}\right)^{3} .
$$

The proof is given on page 96] and is a simple corollary of the discussion and lemmas below. As this theorem is rather incomprehensible, we will investigate what this theorem means for AES-256. The bound obtained by this theorem depends on a parameter $\alpha$. We do not require any specific value $\alpha$ as any $\alpha$ (meeting to the conditions mentioned in Theorem 1) leaves us with a correct bound. For Theorem 1 to give a good bound one must choose a suitable value for the parameter $\alpha$. Choosing large values of $\alpha$ reduces the value of the first term but increases the value of the second term. There seems to be no good closed form for $\alpha$ as these will change with every $q$. The meaning of $\alpha$ will be explained in the proof. We will optimize the parameter $\alpha$ numerically as given in the following corollary.

Corollary 1. For the compression function TANDEM-DM, instantiated e.g. with AES-2561, any adversary asking less than $2^{120.4}$ (backward or forward) oracle queries cannot find a collision with probability greater than 1/2. In this case, $\alpha=$ 24.0 .

\subsection{Proof of Theorem 1}

Analysis Overview. We will analyze if the queries made by the adversary contain the means for constructing a collision of the compression function $F^{T D M}$. Effectively we look to see whether there exist four queries that form a collision (see Figure 2).

To upper bound the probability of the adversary obtaining queries than can be used to construct a collision, we upper bound the probability of the adversary making a query that can be used as the final query to complete such a collision. Namely for each $i, 1 \leq i \leq q$, we upper bound the probability that the answer to the adversary's $i$-th query $\left(K_{i}, X_{i}\right)_{f w d}$ or $\left(K_{i}, Y_{i}\right)_{b w d}$ will allow the adversary to use the $i$-th query to complete the collision. In the latter case, we say that the $i$-th query is 'successful' and we give the attack to the adversary.

As the probability depends naturally on the first $i-1$ queries, we need to make sure that the adversary hasn't already been too lucky with these (or else the probability of the $i$-th query being successful would be hard to upper bound). Concretely, being lucky means, that there exists a large subset of the first $i-1$ queries that all have the same XOR output (see below for a formal definition).

\footnotetext{
${ }^{1}$ Formally, we model the AES-256 block cipher as an ideal block cipher.
} 
Our upper bound thus breaks down into two pieces: an upper bound for the probability of the adversary getting lucky in one defined specific way and the probability of the adversary ever making a successful $i$-th query, conditioned on the fact that the adversary has not yet become lucky by its $(i-1)$-th query.

Analysis Details. Fix numbers $n, q$ and an adversary $\mathcal{A}$ asking $q$ queries to its oracle. We say CoLL ${ }^{T D M}$ if the adversary wins. Note that winning does not necessarily mean finding a collision as will be explained in the following. We upper bound $\operatorname{Pr}\left[\operatorname{ColL}^{T D M}(\mathcal{Q})\right]$ by exhibiting predicates $\operatorname{LuCKY}(\mathcal{Q}), \operatorname{Win} 1(\mathcal{Q})$, $\mathrm{WIN} 2(\mathcal{Q})$ and $\operatorname{Win} 3(\mathcal{Q})$ such that $\operatorname{CoLL}^{T D M}(\mathcal{Q}) \Rightarrow \operatorname{LuCKY}(\mathcal{Q}) \vee \operatorname{Win} 1(\mathcal{Q}) \vee$ Win2 $(\mathcal{Q}) \vee \operatorname{Win} 3(\mathcal{Q})$ and then by upper bounding separately the probabilities $\operatorname{Pr}[\operatorname{Lucky}(\mathcal{Q})], \operatorname{Pr}[\operatorname{Win} 1(\mathcal{Q})], \operatorname{Pr}[\operatorname{Win} 2(\mathcal{Q})]$ and $\operatorname{Pr}[\operatorname{Win} 3(\mathcal{Q})]$. Then, obviously, $\operatorname{Pr}[\operatorname{Colt}(\mathcal{Q})] \leq \operatorname{Pr}[\operatorname{Lucky}(\mathcal{Q})]+\operatorname{Pr}[\operatorname{Win} 1(\mathcal{Q})]+\operatorname{Pr}[\operatorname{Win} 2(\mathcal{Q})]+\operatorname{Pr}[\operatorname{Win} 3(\mathcal{Q})]$ The event $\operatorname{LuCKY}(\mathcal{Q})$ happens if the adversary is lucky, whereas if the adversary is not lucky but makes a successful $i$-th query then one of the other predicates hold.

To state the predicates, we need one additional definition. Let $a(\mathcal{Q})$ be a function defined on query sequences of length $q$ as follows:

$$
a(\mathcal{Q})=\max _{Z \in\{0,1\}^{n}}\left|\left\{i: X_{i} \oplus Y_{i}=Z\right\}\right|
$$

is the maximum size of a set of queries in $\mathcal{Q}$ whose XOR outputs are all the same. The event $\operatorname{Lucky}(\mathcal{Q})$ is now defined by

$$
\operatorname{LUCKY}(\mathcal{Q})=a(\mathcal{Q})>\alpha,
$$

where $\alpha$ is the constant from Theorem 1 (it is chosen depending on $n$ and $q$ by a numerical optimization process). Thus as $\alpha$ is chosen larger $\operatorname{Pr}[\operatorname{Lucky}(\mathcal{Q})]$ diminishes. The other events, $\operatorname{Win} 1(\mathcal{Q}), \operatorname{Win} 2(\mathcal{Q})$ and $\operatorname{Win} 3(\mathcal{Q})$ are different in nature from the event $\operatorname{LuCKY}(\mathcal{Q})$. Simply put, they consider mutually exclusive configurations on how to find a collision for TANDEM-DM (see Figure 2 for an overview).

Notation. As in Figure 2, the four queries that can be used to form a collision will be labeled as TL for the query $\left(X_{i}, K_{i}, Y_{i}\right)$ that is used for the position top left, BL for bottom left, TR for top right and BR for bottom right. Given
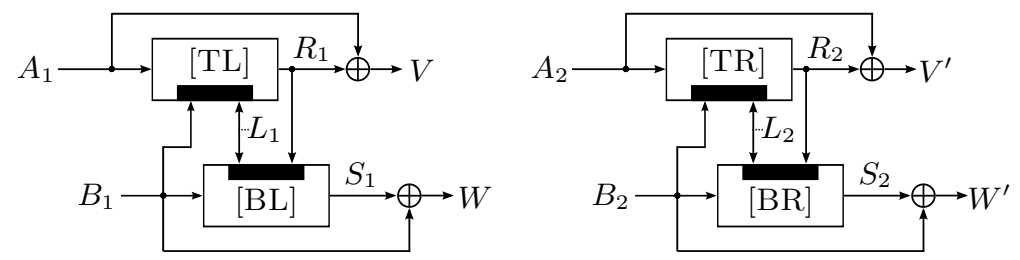

Fig. 2. Generic configuration for a collision, i.e. $V=V^{\prime}$ and $W=W^{\prime}$, for the TANDEM-DM compression function 
$K \in\{0,1\}^{n}$, we will denote the first $a$ bits as $K^{(0 \ldots a-1)} \in\{0,1\}^{a}$ and the leftover bits of $K$ as $K^{(a \ldots n)} \in\{0,1\}^{n-a}$. Furthermore, we say that two queries, e.g. $\mathrm{BL}$ and $\mathrm{BR}$, are equal, i.e. $\mathrm{BL}=\mathrm{BR}$, if and only if there exists an $i$ such that $B L=\left(X_{i}, K_{i}, Y_{i}\right) \in \mathcal{Q}$ and $B R=\left(X_{i}, K_{i}, Y_{i}\right)$.

We will call the configuration necessary for, e.g., predicate $\operatorname{WIN} 1 a(\mathcal{Q})$ simply $1 a$. Now, take for example just this configuration of predicate $\operatorname{WIN} 1 a(\mathcal{Q})$ (i.e. all four queries are different and a collision is found; this case will be defined formally in Definition (3). We say, that the four queries $Q_{i}, Q_{j}, Q_{k}, Q_{l} \in \mathcal{Q}$ fit configuration $1 a$ if and only if

$$
\begin{aligned}
& (i \neq j) \wedge(i \neq k) \wedge(i \neq l) \wedge(j \neq k) \wedge(j \neq l) \wedge(k \neq l) \wedge \\
& \left(X_{i} \oplus Y_{i}=X_{k} \oplus Y_{k}\right) \wedge\left(X_{j} \oplus Y_{j}=X_{l} \oplus Y_{l}\right) \wedge \\
& \left(K_{i}=X_{j} \mid K_{j}^{(0 \ldots n / 2-1)}\right) \wedge\left(K_{j}=K_{i}^{(n / 2 \ldots n-1)} \mid Y_{i}\right) \wedge \\
& \left(K_{k}=X_{l} \mid K_{k}^{(0 \ldots n / 2-1)}\right) \wedge\left(K_{l}=K_{k}^{(n / 2 \ldots n-1)} \mid Y_{k}\right) .
\end{aligned}
$$

We say, that $\operatorname{Fit} 1 a(\mathcal{Q})$ holds if there exist $i, j, k, l \in\{1,2, \ldots, q\}$ such that queries $Q_{i}, Q_{j}, Q_{k}, Q_{l}$ fit configuration $1 a$. The other predicates, namely $\operatorname{Fit} 1 b(\mathcal{Q}), \quad \operatorname{Fit} 1 c(\mathcal{Q}), \quad \operatorname{Fit} 1 d(\mathcal{Q}), \quad \operatorname{Fit} 2 a(\mathcal{Q}), \ldots, \operatorname{Fit} 2 d(\mathcal{Q}), \operatorname{Fit} 3 a(\mathcal{Q}), \ldots$, FIT3d $(\mathcal{Q})$, whose configurations are given in Definition 3, are likewise defined. We also let

$$
\operatorname{Fit} j(\mathcal{Q}):=\operatorname{Fitj} a(\mathcal{Q}) \vee \ldots \vee \operatorname{Fitj} d(\mathcal{Q}) \quad \text { for } \quad j=1,2,3 .
$$

Definition 3. Fit1(Q): The last query is used only once in position TL. Note that this is equal to the case where the last query is used only once in position TR.

Fit $1 a(\mathcal{Q})$ all queries used in the collision are pairwise different,

Fit $1 b(\mathcal{Q}) \mathrm{BL}=\mathrm{TR}$ and $\mathrm{BR}$ is different to $\mathrm{TL}, \mathrm{BL}, \mathrm{TR}$,

Fit $1 c(\mathcal{Q}) \mathrm{BL}=\mathrm{BR}$ and $\mathrm{TR}$ is different to $\mathrm{TL}, \mathrm{BL}, \mathrm{BR}$,

Fit $1 d(\mathcal{Q}) \mathrm{TR}=\mathrm{BR}$ and $\mathrm{BL}$ is different to $\mathrm{TL}$, TR, BR.

Fit2 $(\mathcal{Q}):$ The last query is used only once in position $\mathrm{BL}$. Note that this is equal to the case where the last query is used only once in position BR.

Fit $2 a(\mathcal{Q})$ all queries used in the collision are pairwise different,

Fit $2 b(\mathcal{Q}) \mathrm{TL}=\mathrm{TR}$ and $\mathrm{BR}$ is different to $\mathrm{TL}, \mathrm{BL}, \mathrm{TR}$,

Fit $2 c(\mathcal{Q}) \mathrm{TL}=\mathrm{BR}$ and $\mathrm{TR}$ is different to $\mathrm{TL}, \mathrm{BL}, \mathrm{BR}$,

Fit $2 d(\mathcal{Q}) \mathrm{TR}=\mathrm{BR}$ and $\mathrm{TL}$ is different to $\mathrm{BL}, \mathrm{TR}, \mathrm{BR}$.

$\operatorname{Fit3}(\mathcal{Q}):$ The last query is used twice in a collision.

Fit $3 a(\mathcal{Q})$ last query used in $\mathrm{TL}, \mathrm{BL}(\mathrm{TL}=\mathrm{BL})$ and $\mathrm{TR} \neq \mathrm{BR}$,

Fit $3 b(\mathcal{Q})$ last query used in $\mathrm{TL}, \mathrm{BL}(\mathrm{TL}=\mathrm{BL})$ and $\mathrm{TR}=\mathrm{BR}$,

Fit $3 c(\mathcal{Q})$ last query used in $\mathrm{TL}, \mathrm{BR}(\mathrm{TL}=\mathrm{BR})$ and $\mathrm{BL} \neq \mathrm{TR}$,

Fit3d $(\mathcal{Q})$ last query used in $\mathrm{TL}, \mathrm{BR}(\mathrm{TL}=\mathrm{BR})$ and $\mathrm{BL}=\mathrm{TR}$. 
In Lemma 1 we will show that these configurations cover all possible cases of a collision. We now define the following predicates:

$$
\begin{aligned}
\operatorname{Win} 1(\mathcal{Q}) & =\neg \operatorname{LUCKY}(\mathcal{Q}) \wedge \operatorname{Fit} 1(\mathcal{Q}) \\
\operatorname{Win} 2(\mathcal{Q}) & =\neg(\operatorname{LuCKY}(\mathcal{Q}) \vee \operatorname{Fit} 1(\mathcal{Q})) \wedge \operatorname{FiT} 2(\mathcal{Q}), \\
\operatorname{Win} 3(\mathcal{Q}) & =\neg(\operatorname{LuckY}(\mathcal{Q}) \vee \operatorname{Fit} 1(\mathcal{Q}) \vee \operatorname{FiT} 2(\mathcal{Q})) \wedge \operatorname{FiT} 3(\mathcal{Q}) .
\end{aligned}
$$

Thus Win3 $(\mathcal{Q})$, for example, is the predicate which is true if and only if $a(\mathcal{Q}) \leq \alpha$ (i.e. $\neg \operatorname{LUCKY}(\mathcal{Q})$ ) and $\mathcal{Q}$ contains queries that fit configurations $3 a, 3 b, 3 c$ or $3 d$ but $\mathcal{Q}$ does not contain queries fitting configurations $1 a, \ldots, 1 d, 2 a, \ldots 2 d$. We now show, that $\operatorname{CoLL}^{T D M}(\mathcal{Q}) \Longrightarrow \operatorname{LUCKY}(\mathcal{Q}) \vee \operatorname{Win} 1(\mathcal{Q}) \vee W i n 2(\mathcal{Q}) \vee \operatorname{Win} 3(\mathcal{Q})$.

\section{Lemma 1}

$$
\operatorname{CoLL}^{T D M}(\mathcal{Q}) \Longrightarrow \operatorname{LUCKY}(\mathcal{Q}) \vee \operatorname{Win} 1(\mathcal{Q}) \vee \operatorname{Win} 2(\mathcal{Q}) \vee \operatorname{Win} 3(\mathcal{Q})
$$

Proof. If the adversary is not lucky, i.e. $\neg \operatorname{LUCKY}(\mathcal{Q})$, then

$$
\operatorname{Fit} 1 a(\mathcal{Q}) \vee \ldots \vee \operatorname{FiT} 3 d(\mathcal{Q}) \Longrightarrow \operatorname{Win} 1 a(\mathcal{Q}) \vee \ldots \mathrm{W} \operatorname{In} 3 d(\mathcal{Q})
$$

holds. So it is sufficient to show that $\operatorname{CoLL}^{T D M}(\mathcal{Q}) \Longrightarrow \operatorname{FiT} 1 a(\mathcal{Q}) \vee \ldots \vee$ $\operatorname{FiT} 3 d(\mathcal{Q})$. Now, say $\operatorname{ColL}^{T D M}(\mathcal{Q})$ and $\neg \operatorname{LUCKY}(\mathcal{Q})$. Then a collision can be constructed from the queries $\mathcal{Q}$. That is, our query history $\mathcal{Q}$ contains queries $Q_{i}, Q_{j}, Q_{k}, Q_{l}$ (see Figure 2) such that we have a collision, i.e. $V=V^{\prime}$ and $W=W^{\prime}$ and $\mathrm{TL} \neq \mathrm{TR}$. Note that the last condition suffices to ensure a real collision (a collision from two different inputs).

First assume that the last query is used once in the collision. If it is used in position TL, then we have to consider the queries BL, TR and BR. If these three queries are all different (and as the last query is only used once), then $\operatorname{FiT} 1 a(\mathcal{Q})$. If $\mathrm{BL}=\mathrm{TR}$ and $\mathrm{BR}$ is different, then $\operatorname{FiT} 1 b(\mathcal{Q})$. If $\mathrm{BL}=\mathrm{BR}$ and $\mathrm{TR}$ is different, then $\operatorname{FiT} 1 c(\mathcal{Q})$. If $\mathrm{TR}=\mathrm{BR}$ and BL is different, then $\operatorname{FiT} 1 d(\mathcal{Q})$. If $\mathrm{BL}=\mathrm{TR}=\mathrm{BR}$, then we have $\mathrm{BL}=\mathrm{BR}$ and $\mathrm{TL}=\mathrm{TR}$ and this would not result in a collision since the inputs to the two compression functions would be the same. As no cases are left, we are done (for the case that the last query is used only in position TL).

If the last query is used once in the collision and is used in position BL, then we have to consider the queries TL, TR and BR. If these three queries are all different (and as the last query is only used once), then Fit2a $(\mathcal{Q})$. If $\mathrm{TL}=\mathrm{TR}$ and $\mathrm{BR}$ is different, then $\operatorname{FrT} 2 b(\mathcal{Q})$. If $\mathrm{TL}=\mathrm{BR}$ and $\mathrm{TR}$ is different, then FiT $2 c(\mathcal{Q})$. If $\mathrm{TR}=\mathrm{BR}$ and $\mathrm{TL}$ is different, then FiT2 $(\mathcal{Q})$. If $\mathrm{TL}=\mathrm{TR}=\mathrm{BR}$, it follows $\mathrm{TL}=\mathrm{TR}$ and $\mathrm{BL}=\mathrm{BR}$ and this would not result in a collision since the inputs to the two compression functions would be the same. As no cases are left, we are done.

We now analyze the case when the last query is used twice in the collision. First, assume that the query is used for the positions TL and BL (TL $=\mathrm{BL}$ ).

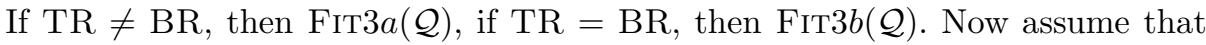
the query is employed for the pair TL and BR $(\mathrm{TL}=\mathrm{BR})$. Note, that this case 
is equal to the case where the query is employed for $\mathrm{BL}$ and $\mathrm{TR}$. If $\mathrm{BL} \neq \mathrm{TR}$, then $\operatorname{FiT} 3 c(\mathcal{Q})$, if $\mathrm{BL}=\mathrm{TR}$, then $\operatorname{FiT} 3 d(\mathcal{Q})$. The other cases, i.e. the last query is employed either for $\mathrm{TL}=\mathrm{TR}$ or $\mathrm{BL}=\mathrm{BR}$, do not lead to a real collision as this would imply the same compression function input. As no cases are left, we are done.

If the last query is used more than twice for the collision we do not get a real collision as this case would imply either $\mathrm{TL}=\mathrm{TR}$ or $\mathrm{BL}=\mathrm{BR}$ and we have the same input, again, for both compression functions.

The next step is to upper bound the probability of the predicates $\operatorname{LuCKY}(\mathcal{Q})$, $\operatorname{Win} 1(\mathcal{Q}), \operatorname{Win} 2(\mathcal{Q})$ and $\operatorname{Win} 3(\mathcal{Q})$.

Lemma 2. Let $\alpha$ be as in Theorem 1. If $\alpha>e$ and $\tau=N^{\prime} \alpha / q$, then

$$
\operatorname{Pr}[\operatorname{LUCKY}(\mathcal{Q})] \leq q 2^{n} e^{\tau \nu(1-\ln \tau)} .
$$

The proof is quite technical and is given in Appendix A.

Lemma 3. $\operatorname{Pr}[\operatorname{Win} 1(\mathcal{Q})] \leq q \alpha / N^{\prime}+2 q /\left(N^{\prime}\right)^{2}+q /\left(N^{\prime}\right)^{3}$.

Proof. As $\operatorname{Win} 1(\mathcal{Q})=\neg \operatorname{LuCKY}(\mathcal{Q}) \wedge \operatorname{Fit} 1(\mathcal{Q})$, we will upper bound the probabilities of $\operatorname{FiT} 1 a(\mathcal{Q}), \operatorname{FiT} 1 b(\mathcal{Q}), \operatorname{FIT} 1 c(\mathcal{Q})$ and $\operatorname{FiT} 1 d(\mathcal{Q})$ separately in order to get an upper bound for $\operatorname{Pr}[\operatorname{FiT} 1(\mathcal{Q})] \leq \operatorname{FiT} 1 a(\mathcal{Q})+\ldots+\operatorname{FiT} 1 d(\mathcal{Q})$. We will use the notations given in Figure 3 .
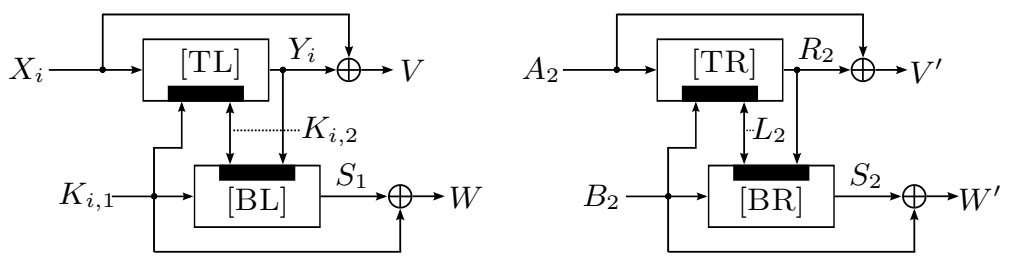

Fig. 3. Notations used for $\operatorname{Win} 1(\mathcal{Q})$

Let $\mathcal{Q}_{i}$ denote the first $i$ queries made by the adversary. The term 'last query' means the latest query made by the adversary (we examine the adversary's queries $\left(K_{i}, X_{i}\right)_{f w d}$ or $\left(K_{i}, Y_{i}\right)_{b w d}$ one at a time as they come in). The last query is always given index $i$. We say the last query is successful if the output $X_{i}$ or $Y_{i}$ for the last query is such that $a\left(\mathcal{Q}_{i}\right)<\alpha$ and such that the adversary can use the query $\left(X_{i}, K_{i}, Y_{i}\right)$ to fit the configuration given in Figure 3 using only queries in $\mathcal{Q}_{i}$ (in particular, the last query must be used once in the fitting for that query to count as successful). The goal is thus to upper bound the adversary's chance of ever making a successful last query. The basic setup for upper bounding the probability of success in a given case is to upper bound the maximum number of different outputs $Y_{i}$ or $X_{i}$ (depending on whether the last query is a forward or a backward query) that would allow the query $\left(X_{i}, K_{i}, Y_{i}\right)$ to be used to fit 
the configuration, and then divide this number by $N^{\prime}=2^{n}-q$ (since either $Y_{i}$ or $X_{i}$, depending, is chosen randomly among a set of at least $2^{n}-q$ different values). The ratio is then multiplied by $q$, since the adversary makes $q$ queries in all, each of which could become a successful last query.

(i) Fit $1 a(\mathcal{Q})$ : The last query, wlog. $\left(X_{i}, K_{i, 1} \mid K_{i, 2}, Y_{i}\right)$, is used in position TL. We do not care whether the last query was a forward or backward query since the analysis below is the same. All queries are, as claimed, pairwise different. We give the adversary for free the answer to the forward query BL, $\left(K_{i, 1}, K_{i, 2} \mid Y_{i}, S_{1}\right)$. Then we have $V=Y_{i} \oplus X_{i}$ and $W=S_{1} \oplus K_{i, 1}$. This pair of queries is successful if the adversary's query history $\mathcal{Q}_{i-1}$ contains a pair $\left(A_{2}, B_{2} \mid L_{2}, R_{2}\right),\left(B_{2}, L_{2} \mid R_{2}, S_{2}\right)$ such that $V=X_{i} \oplus Y_{i}=R_{2} \oplus A_{2}=V^{\prime}$ and $W=S_{1} \oplus K_{i, 1}=S_{2} \oplus B_{2}=W^{\prime}$. There are at most $\alpha$ queries in $\mathcal{Q}_{i-1}$ that can possibly be used for query in TR that all lead to a collision in the top row, i.e. $V=V^{\prime}$. Therefore we have at most $\alpha$ possibilities for the query in BR since the query in TR uniquely determines the query BR. Thus, the last query has a chance of $\leq \alpha / N^{\prime}$ of succeeding. So the total chance of making a successful query of this type is $\leq q \alpha / N^{\prime}$.

(ii) $\operatorname{FrT1} b(\mathcal{Q})$ : Again, the last query, wlog. $\left(X_{i}, K_{i, 1} \mid K_{i, 2}, Y_{i}\right)$, is used in position TL. We give the adversary for free the answer to the forward query BL, $\left(K_{i, 1}, K_{i, 2} \mid Y_{i}, S_{1}\right)$. By our claim, as $\mathrm{BL}=\mathrm{TR}$, we have $A_{2}=K_{i, 1}, B_{2}=$ $K_{i, 2}, L_{2}=Y_{i}$ and $R_{2}=S_{1}$. It follows that for any given query $i$ for TL, we have at most one query for TR to form a collision $V=V^{\prime}$ (as the query TL uniquely determines the query BL and the queries BL and TR are equal) and therefore have at most one query BR in our query history to form a collision $W=W^{\prime}$. The last query has a chance of $\leq 1 /\left(N^{\prime} \cdot N^{\prime}\right)$ of succeeding and so the total chance of making a successful query in the attack is $\leq q /\left(N^{\prime}\right)^{2}$.

(iii) $\operatorname{FIT} 1 c(\mathcal{Q})$ : As this analysis is essentially the same as for $\operatorname{Fit} 1 b(\mathcal{Q})$ we conclude with a total chance of success for this type of query of $\leq q /\left(N^{\prime}\right)^{2}$.

(iv) FIT1d( $\mathcal{Q})$ : Again, the last query, wlog. $\left(X_{i}, K_{i, 1} \mid K_{i, 2}, Y_{i}\right)$, is used in position TL. We give the adversary for free the answer to the forward query BL, $\left(K_{i, 1}, K_{i, 2} \mid Y_{i}, S_{1}\right)$. Note, that this query is trivially different from the query in TL as we assume that the last query is only used once in this configuration (the case in which the two queries, TL and BL, are equal is discussed in the analysis of $\operatorname{Win} 3(\mathcal{Q}))$. We have $V=Y_{i} \oplus X_{i}$ and $W=S_{1} \oplus K_{i, 1}$. As by our claim, we assume $\mathrm{TR}=\mathrm{BR}$. The pair of queries for TL and $\mathrm{BL}$ is successful if the adversary's query history $\mathcal{Q}_{i-1}$ contains a query $\left(A_{2}, B_{2} \mid L_{2}, R_{2}\right)$ such that $V=R_{2} \oplus A_{2}=V^{\prime}$ and $W=R_{2} \oplus A_{2}=W^{\prime}$, i.e. $V=W=V^{\prime}=W^{\prime}$. Moreover, it follows from $B_{2}=R_{2}=L_{2}$ that $V=W=V^{\prime}=W^{\prime}=0$. As at least three of them are chosen randomly by the initial query input (wlog. $\left.V, W, V^{\prime}\right)$, the query has a chance of success in the $i$-th query $\leq 1 /\left(N^{\prime} \cdot N^{\prime} \cdot N^{\prime}\right)$ and therefore a total chance of success $\leq q /\left(N^{\prime}\right)^{3}$.

The claim follows by adding up the individual results. 
Lemma 4. $\operatorname{Pr}[\operatorname{Win} 2(\mathcal{Q})] \leq q \alpha / N^{\prime}+2 q /\left(N^{\prime}\right)^{2}+q /\left(N^{\prime}\right)^{3}$.

As the proof and the result is (in principle) identical to the proof of $\operatorname{Pr}[\operatorname{Win} 1(\mathcal{Q})$ ] we omitted the details of the proof.

Lemma 5. $\operatorname{Pr}[\operatorname{Win} 3(\mathcal{Q})] \leq 2 q \alpha / N^{\prime}+2 q /\left(N^{\prime}\right)^{2}$.

Proof. The same notations and preliminaries as in the proof of Lemma 3 are used.

(i) $\mathrm{W} \operatorname{In} 3 a(\mathcal{Q})$ : The last query, wlog. $\left(X_{i}, K_{i, 1} \mid K_{i, 2}, Y_{i}\right)$ is used in positions TL and BL. We do not care whether the last query is a forward or backward query since the analysis is the same. It follows, that $X_{i}=K_{i, 1}=K_{i, 2}=Y_{i}$ and therefore $V=X_{i} \oplus Y_{i}=W=0$. We assume that the adversary is successful concerning these restraints, i.e. has found a query TL that can also be used for BL such as $X_{i}=Y_{i}=K_{i, 1}=K_{i, 2}$. (Note, that this condition is quite hard.) We do have at most $\alpha$ queries in $\mathcal{Q}_{i-1}$ that can possibly be used for a query in TR and that lead to a collision in the top row, i.e. $0=V=V^{\prime}$. For every such query $\mathrm{TR}$ we have at most one corresponding query in $\mathcal{Q}_{i-1}$ that can be used in position BR. So the last query has a chance of $\leq \alpha / N^{\prime}$ of succeeding and so the total chance of making a successful query of this type during the attack is $\leq q \alpha / N^{\prime}$.

(ii) $\operatorname{WIn} 3 b(\mathcal{Q})$ : The last query, wlog. $\left(X_{i}, K_{i, 1} \mid K_{i, 2}, Y_{i}\right)$ is used in positions TL and BL. We do not care whether the last query is a forward or backward query since the analysis is the same. It follows, that $X_{i}=K_{i, 1}=K_{i, 2}=Y_{i}$ and therefore $V=X_{i} \oplus Y_{i}=W=0$. We assume again that the adversary is successful concerning these restraints, i.e. has found a query TL that can also be used for BL. We do have at most $\alpha$ queries in $\mathcal{Q}_{i-1}$ that can possibly be used for a query in TR and that lead to a collision in the top row, i.e. $0=V=V^{\prime}$. We assume that we can use any such query equally as the corresponding query for BR. In reality, this gives the adversary with high probability more power than he will have. Thus, the last query has a chance of $\leq \alpha / N^{\prime}$ of succeeding and so the total chance of making a successful query of this type during the attack is $\leq q \alpha / N^{\prime}$. As discussed above, this upper bound is likely to be generous.

(iii) $\operatorname{WIN} 3 c(\mathcal{Q})$ : The last query, wlog. $\left(X_{i}, K_{i, 1} \mid K_{i, 2}, Y_{i}\right)$ is used in positions TL and BR. Note, that this situation is equal to the last query being used in position BL and TR. We do not care whether the last query is a forward or backward query. We give the adversary for free the answer to the forward query BL, $\left(K_{i, 1}, K_{i, 2} \mid Y_{i}, S_{1}\right)$. We also give the adversary for free the answer to the backward query TR, $\left(A_{2}, X_{i} \mid K_{i, 1}, K_{i, 2}\right)$. The probability for the $i$-th query to be successful is equal to $\operatorname{Pr}\left[V=V^{\prime}\right] \cdot \operatorname{Pr}\left[W=W^{\prime}\right]$, and as $W$ and $V^{\prime}$ are guaranteed to be chosen independently and randomly the chance of success is $\leq 1 /\left(N^{\prime}\right)^{2}$. The total chance of success is therefore $\leq q /\left(N^{\prime}\right)^{2}$.

(iv) $\mathrm{W} \operatorname{In} 3 d(\mathcal{Q})$ : The last query, wlog. $\left(X_{i}, K_{i, 1} \mid K_{i, 2}, Y_{i}\right)$ is used in positions TL and BR. Note, that this situation is equal to the last query being used in position $\mathrm{BL}$ and TR. We do not care whether the last query is a forward or 
backward query. We give the adversary for free the answer to the forward query BL, $\left(K_{i, 1}, K_{i, 2} \mid Y_{i}, S_{1}\right)$. (This query is also used for position TR and it follows (by comparing the input values of query BL that is used for TR with them of BR) $K_{i, 2}\left|Y_{i}=X_{i}\right| K_{i, 1}$ and $S_{1}=K_{i, 2}$. Comparing the outputs we get a collision in the top row of the compression functions $\operatorname{Pr}\left[V=V^{\prime}\right]=$ $\operatorname{Pr}\left[E_{K_{i, 1} \mid K_{i, 2}}\left(X_{i}\right) \oplus X_{i}=E_{K_{i, 2} \mid Y_{i}}\left(K_{i, 1}\right) \oplus K_{i, 1}\right]$, where $Y_{i}=E_{K_{i, 1} \mid K_{i, 2}}$, with probability $\leq 1 / N^{\prime}$. This is, because the input values $X_{i}, K_{i, 1}, K_{i, 2}$ have to be in such a way that the two inputs to the $E$ oracle are different (if they are not, we would have no colliding inputs for the two compression functions). For the bottom row of the compression function we get, similarly, a collision with probability $\leq 1 / N^{\prime}$. So the total chance for succeeding is in this case $\leq q /\left(N^{\prime}\right)^{2}$ as we have again at most $q$ queries by the adversary.

We now give the proof for Theorem 1

Proof. (of Theorem 1)

The proof follows directly with Lemma 1, 2, 3, 4, and Lemma 5.

\section{Preimage Resistance}

Although, the main focus is on collision resistance, we are also interested in the difficulty of inverting the compression function of TANDEM-DM. Generally speaking, second-preimage resistance is a stronger security requirement than preimage resistance. A preimage may have some information of another preimage which produces the same output. However, in the ideal cipher model, for the compression function TANDEM-DM, a second-preimage has no information useful to find another preimage. Thus, only preimage resistance is analyzed. Note, that there have be various results that discuss attacks on iterated hash functions in terms of pre- and second-preimage, e.g. long-message second-preimage attacks [6, 16], in such a way that the preimage-resistance level cannot easily be transferred to an iterated hash function built on it.

The adversary's goal is to output a preimage $(G, H, M)$ for a given $\sigma$, where $\sigma$ is taken randomly from the output domain, such as $F(G, H, M)=\sigma$. As in the proof of Theorem 1 we will again dispense the adversary from having to output such a preimage. We will determine whether the adversary has been successful or not by examining its query history $\mathcal{Q}$. We say, that $\operatorname{PrEImG}(\mathcal{Q})$ holds if there is such a preimage and $\mathcal{Q}$ contains all the queries necessary to compute it.

Definition 4. (Inverting random points) Let $F$ be a block cipher based compression function, $F:\{0,1\}^{3 n} \rightarrow\{0,1\}^{2 n}$. Fix an adversary $\mathcal{A}$ that has access to oracles $E, E^{-1}$. Then the advantage of $\mathcal{A}$ of inverting $F$ is the real number

$$
\begin{aligned}
\operatorname{Adv}_{F}^{\operatorname{INv}}(\mathcal{A}) & =\operatorname{Pr}\left[E \stackrel{R}{\leftarrow} \operatorname{BC}(n, k) ; \sigma \stackrel{R}{\leftarrow}\{0,1\}^{2 n}:\right. \\
(G, H, M) & \left.\stackrel{R}{\leftarrow} \mathcal{A}^{E, E^{-1}}(\sigma): F(G, H, M)=\sigma\right] .
\end{aligned}
$$

Again, for $q \geq 1$, we write 


$$
\mathbf{A d v}_{F}^{\mathrm{INv}}(q)=\max _{\mathcal{A}}\left\{\mathbf{A d v}_{F}^{\mathrm{INv}}(\mathcal{A})\right\}
$$

where the maximum is taken over all adversaries that ask at most $q$ oracle queries. Note that there has been a discussion on formalizations of preimage resistance. For details we refer to [2, Section 2, Appendix B].

\subsection{Preimage Security}

The preimage resistance of the compression function TANDEM-DM is given in the following Theorem.

Theorem 2. Let $F:=F^{T D M}$ be as in Definition [1, For every $N^{\prime}=2^{n}-q$ and $q>1$

$$
\operatorname{Adv}_{F}^{\text {INv }}(q) \leq 2 q /\left(N^{\prime}\right)^{2} .
$$

Proof. Fix $\sigma=\left(\sigma_{1}, \sigma_{2}\right) \in\{0,1\}^{2 n}$ where $\sigma_{1}, \sigma_{2} \in\{0,1\}^{n}$ and an adversary $\mathcal{A}$ asking $q$ queries to its oracles. We upper bound the probability that $\mathcal{A}$ finds a preimage for a given $\sigma$ by examining the oracle queries as they come in and upper bound the probability that the last query can be used to create a preimage, i.e. we upper bound $\operatorname{Pr}[\operatorname{PreImG}(\mathcal{Q})]$. Let $\mathcal{Q}_{i}$ denote the first $i$ queries made by the adversary. The term 'last query' means the latest query made by the adversary since we examine again the adversary's queries $\left(K_{i}, X_{i}\right)_{f w d}$ or $\left(K_{i}, X_{i}\right)_{b w d}$ one at a time as they come in. The last query is always given index $i$.

Case 1: The last query $\left(X_{i}, K_{i}, Y_{i}\right)$ is used in the top row. Either $X_{i}$ or $Y_{i}$ was randomly assigned by the oracle from a set of at least the size $N^{\prime}$. The query is successful in the top row if $X_{i} \oplus Y_{i}=\sigma_{1}$ and thus has a chance of success of $\leq 1 / N^{\prime}$. In $\mathcal{Q}_{i}$ there is at most one query $Q_{j}$ that matches for the bottom row. If there is no such query in $\mathcal{Q}_{i}$ we give this query $Q_{j}$ the adversary for free. This 'bottom' query is successful if $X_{j} \oplus Y_{j}=\sigma_{2}$ and therefore has a chance of success of $\leq 1 / N^{\prime}$. So the total chance of success after $q$ queries is $\leq q /\left(N^{\prime}\right)^{2}$.

Case 2: The last query $\left(X_{i}, K_{i}, Y_{i}\right)$ is used in the bottom row. The analysis is essentially the same as in Case 1 . The total chance of success is $\leq q /\left(N^{\prime}\right)^{2}$, too.

As any query can either be used in the top or in the bottom row, the claim follows.

\section{Discussion and Conclusion}

In this paper, we have investigated the security of TANDEM-DM, a long outstanding DBL compression function based on an $(n, 2 n)$ block cipher. In the ideal cipher model, we showed that this construction has birthday-type collision resistance. As there are some generous margins in the proof it is likely, that 
TANDEM-DM is even more secure. Our bound for preimage resistance is far from optimal, but we have not found an attack that would classify this bound as tight.

Somewhat surprisingly, there seems to be only one practical rate $1 / 2 \mathrm{DBL}$ compression function that also has a birthday-type security guarantee. It was presented at FSE'06 by Hirose [13]. Taking into account that it was presented about 15 years after TANDEM-DM, it is clear that there needs still to be a lot of research done in the field of block cipher based hash functions, e.g. there are still security proofs missing for the aforementioned ABREAST-DM and MDC-4 compression or hash functions.

\section{Acknowledgments}

The authors wish to thank the anonymous reviewers for helpful comments.

\section{References}

1. ANSI. ANSI X9.31:1998: Digital Signatures Using Reversible Public Key Cryptography for the Financial Services Industry (rDSA). American National Standards Institute (1998)

2. Black, J., Rogaway, P., Shrimpton, T.: Black-Box Analysis of the Block-CipherBased Hash-Function Constructions from PGV. In: Yung, M. (ed.) CRYPTO 2002. LNCS, vol. 2442, pp. 320-335. Springer, Heidelberg (2002)

3. Meyer, C., Matyas, S.: Secure program load with manipulation detection code (1988)

4. Coppersmith, D., Pilpel, S., Meyer, C.H., Matyas, S.M., Hyden, M.M., Oseas, J., Brachtl, B., Schilling, M.: Data authentication using modification dectection codes based on a public one way encryption function. U.S. Patent No. 4, 908, 861, March $13(1990)$

5. Cramer, R. (ed.): EUROCRYPT 2005. LNCS, vol. 3494. Springer, Heidelberg (2005)

6. Dean, R.D.: Formal aspects of mobile code security. PhD thesis, Princeton, NJ, USA (1999); Adviser-Andrew Appel

7. den Boer, B., Bosselaers, A.: Collisions for the Compression Function of MD5. In: Helleseth, T. (ed.) EUROCRYPT 1993. LNCS, vol. 765, pp. 293-304. Springer, Heidelberg (1993)

8. Freitag, E., Busam, R.: Complex Analysis, 1st edn. Springer, Heidelberg (2005)

9. Even, S., Mansour, Y.: A Construction of a Cipher From a Single Pseudorandom Permutation. In: Imai, H., Rivest, R.L., Matsumoto, T. (eds.) ASIACRYPT 1991. LNCS, vol. 739, pp. 210-224. Springer, Heidelberg (1991)

10. Dobbertin, H.: The status of MD5 after a recent attack (1996)

11. Hattori, M., Hirose, S., Yoshida, S.: Analysis of Double Block Length Hash Functions. In: Paterson, K.G. (ed.) Cryptography and Coding 2003. LNCS, vol. 2898, pp. 290-302. Springer, Heidelberg (2003)

12. Hirose, S.: Provably Secure Double-Block-Length Hash Functions in a Black-Box Model. In: Park, C., Chee, S. (eds.) ICISC 2004. LNCS, vol. 3506, pp. 330-342. Springer, Heidelberg (2004) 
13. Hirose, S.: Some Plausible Constructions of Double-Block-Length Hash Functions. In: Robshaw, M.J.B. (ed.) FSE 2006. LNCS, vol. 4047, pp. 210-225. Springer, Heidelberg (2006)

14. Hohl, W., Lai, X., Meier, T., Waldvogel, C.: Security of Iterated Hash Functions Based on Block Ciphers. In: Stinson [36], pp. 379-390

15. ISO/IEC. ISO DIS 10118-2: Information technology - Security techniques - Hashfunctions, Part 2: Hash-functions using an n-bit block cipher algorithm. First released in 1992 (2000)

16. Kelsey, J., Schneier, B.: Second Preimages on n-Bit Hash Functions for Much Less than $2^{\mathrm{n}}$ Work. In: Cramer [5], pp. 474-490

17. Kilian, J., Rogaway, P.: How to Protect DES Against Exhaustive Key Search. In: Koblitz, N. (ed.) CRYPTO 1996. LNCS, vol. 1109, pp. 252-267. Springer, Heidelberg (1996)

18. Knudsen, L.R., Lai, X., Preneel, B.: Attacks on Fast Double Block Length Hash Functions. J. Cryptology 11(1), 59-72 (1998)

19. Knudsen, L.R., Muller, F.: Some Attacks Against a Double Length Hash Proposal. In: Roy, B.K. (ed.) ASIACRYPT 2005. LNCS, vol. 3788, pp. 462-473. Springer, Heidelberg (2005)

20. Lai, X., Massey, J.L.: Hash Function Based on Block Ciphers. In: Rueppel, R.A. (ed.) EUROCRYPT 1992. LNCS, vol. 658, pp. 55-70. Springer, Heidelberg (1992)

21. Lucks, S.: A Collision-Resistant Rate-1 Double-Block-Length Hash Function. In: Biham, E., Handschuh, H., Lucks, S., Rijmen, V. (eds.) Symmetric Cryptography, Internationales Begegnungs- und Forschungszentrum fuer Informatik (IBFI), Schloss Dagstuhl, Germany. Dagstuhl Seminar Proceedings, vol. 07021 (2007)

22. Rabin, M.: Digitalized Signatures. In: DeMillo, R., Dobkin, D., Jones, A., Lipton, R. (eds.) Foundations of Secure Computation, pp. 155-168. Academic Press, London (1978)

23. Menezes, A., van Oorschot, P.C., Vanstone, S.A.: Handbook of Applied Cryptography. CRC Press, Boca Raton (1996)

24. Merkle, R.C.: One Way Hash Functions and DES. In: Brassard, G. (ed.) CRYPTO 1989. LNCS, vol. 435, pp. 428-446. Springer, Heidelberg (1989)

25. Nandi, M., Lee, W.I., Sakurai, K., Lee, S.-J.: Security Analysis of a 2/3-Rate Double Length Compression Function in the Black-Box Model. In: Gilbert, H., Handschuh, H. (eds.) FSE 2005. LNCS, vol. 3557, pp. 243-254. Springer, Heidelberg (2005)

26. NIST National Institute of Standards and Technology. FIPS 180-1: Secure Hash Standard (April 1995), http://csrc.nist.gov

27. NIST National Institute of Standards and Technology. FIPS 180-2: Secure Hash Standard (April 1995), http://csrc.nist.gov

28. Preneel, B., Govaerts, R., Vandewalle, J.: Hash Functions Based on Block Ciphers: A Synthetic Approach. In: Stinson [36], pp. 368-378

29. Rivest, R.L.: RFC 1321: The MD5 Message-Digest Algorithm. Internet Activities Board (April 1992)

30. Rivest, R.L.: The MD4 Message Digest Algorithm. In: Menezes, A., Vanstone, S.A. (eds.) CRYPTO 1990. LNCS, vol. 537, pp. 303-311. Springer, Heidelberg (1990)

31. Rogaway, P., Steinberger, J.P.: Constructing Cryptographic Hash Functions from Fixed-Key Blockciphers. In: Wagner, D. (ed.) CRYPTO 2008. LNCS, vol. 5157, pp. 433-450. Springer, Heidelberg (2008)

32. Rogaway, P., Steinberger, J.P.: Security/Efficiency Tradeoffs for PermutationBased Hashing. In: Smart, N.P. (ed.) EUROCRYPT 2008. LNCS, vol. 4965, pp. 220-236. Springer, Heidelberg (2008) 
33. Satoh, Haga, Kurosawa: Towards Secure and Fast Hash Functions. TIEICE: IEICE Transactions on Communications/Electronics/Information and Systems (1999)

34. Steinberger, J.P.: The collision intractability of mdc-2 in the ideal cipher model. IACR ePrint Archive, Report 2006/294 (2006), http://eprint.iacr.org/2006/294

35. Steinberger, J.P.: The Collision Intractability of MDC-2 in the Ideal-Cipher Model. In: Naor, M. (ed.) EUROCRYPT 2007. LNCS, vol. 4515, pp. 34-51. Springer, Heidelberg (2007)

36. Stinson, D.R. (ed.): CRYPTO 1993. LNCS, vol. 773. Springer, Heidelberg (1994)

37. Wang, X., Lai, X., Feng, D., Chen, H., Yu, X.: Cryptanalysis of the Hash Functions MD4 and RIPEMD. In: Cramer [5], pp. 1-18

38. Wang, X., Yin, Y.L., Yu, H.: Finding Collisions in the Full SHA-1. In: Shoup, V. (ed.) CRYPTO 2005. LNCS, vol. 3621, pp. 17-36. Springer, Heidelberg (2005)

\section{A Proof of Lemma 2}

Note that this proof is essentially due to Steinberger 34]. We can rephrase the problem of upper bounding $\operatorname{Pr}[\operatorname{LUCKY}(\mathcal{Q})]=\operatorname{Pr}[a(\mathcal{Q})>\alpha]$ as a balls-in-bins question. Let $N=2^{n}$ be the number of bins and $q$ be the number of balls to be thrown. The $i$-th ball falls into the $j$-th bin if the XOR output of the $i$-th query is equal to the XOR output of the $j$-th query, i.e. $X_{i} \oplus Y_{i}=X_{j} \oplus Y_{j}$. In the following we will upper bound the probability that some bin contains more than $\alpha$ balls. As the balls are thrown independent of each other, the $i$-th ball always has probability $\leq p=1 /\left(2^{n}-q\right)$ of falling in the $j$-th bin. This is because the XOR output of the $i$-th query is chosen uniformly at random from a set of size at least $2^{n}-q$. If we let $B(k)$ be the probability of having exactly $k$ balls in a particular bin, say bin 1 , then

$$
B(k) \leq p^{k}\left(\begin{array}{l}
q \\
k
\end{array}\right) .
$$

Let $\nu=q p$, where $\nu$ is an upper bound for the expected number of balls in any bin. By Stirlings approximation [8] (and $e^{x}$ being the exponential function)

$$
n ! \leq \sqrt{2 \pi n} \cdot\left(\frac{n}{e}\right)^{n} \cdot e^{1 /(12 n)}
$$

we can upper bound $B(k)$ as follows:

$$
\begin{aligned}
B(k) & \leq p^{k} \frac{q !}{k !(q-k) !} \\
& \leq \frac{p^{k}}{\sqrt{2 \pi}} \sqrt{\frac{q}{k(q-k)}} \cdot \frac{q^{q}}{k^{k}(q-k)^{1-k}} \cdot \frac{e^{k} \cdot e^{q-k}}{e^{q}} \cdot e^{\frac{1}{12}(q-k-(q-k))} \\
& \leq k^{-k} \nu^{k}\left(\frac{q}{q-k}\right) \\
& \leq \nu^{k} \cdot k^{-k} \cdot e^{k} .
\end{aligned}
$$


Since $\alpha=\tau \nu$ we get

$$
B(\alpha) \leq \frac{\nu^{\tau \nu} e^{\tau \nu}}{(\tau \nu)^{\tau \nu}}=\frac{e^{\tau \nu}}{\tau^{\tau \nu}}=e^{\tau \nu(1-\ln \tau)} .
$$

As $B(\alpha)$ is a decreasing function of $\alpha$ if $(1-\ln \tau)<0$, it follows that $B(\alpha)$ is a decreasing function if $\alpha>e$. And so we have

$$
\begin{aligned}
\operatorname{Pr}[a(\mathcal{Q})>\alpha] & \leq 2^{n} \cdot \sum_{j=\alpha}^{q} B(j) \\
& \leq q 2^{n} B(\alpha) \leq q 2^{n} e^{\tau \nu(1-\ln \tau)} .
\end{aligned}
$$

This proves our claim.

\section{B Security of the FSE'06 Proposal by Hirose for a DBL Compression Function}

At FSE'06, Hirose [13] proposed a DBL compression function (Definition 5 and Figure 41). He proved that when his compression function $F^{\text {Hirose }}$ is employed in an iterated hash function $H$, then no adversary asking less than $2^{125.7}$ queries can have more than a chance of 0.5 in finding a collision for $n=128$. As he has not stated a security result for the compression function we do here for comparison with TANDEM-DM.

\section{B.1 Compression Function}

Definition 5. Let $F^{\text {Hirose }}:\{0,1\}^{2 n} \times\{0,1\}^{n} \rightarrow\{0,1\}^{2 n}$ be a compression function such that $\left(G_{i}, H_{i}\right)=F^{\text {Hirose }}\left(G_{i-1}, H_{i-1}, M_{i}\right)$ where $G_{i}, H_{i}, M_{i} \in\{0,1\}^{n}$. $F^{\text {Hirose }}$ is built upon a $(n, 2 n)$ block cipher $E$ as follows:

$$
\begin{aligned}
G_{i} & =\mathrm{F}_{T}\left(G_{i-1}, H_{i-1}, M_{i}\right)=E\left(G_{i-1}, H_{i-1} \mid M_{i}\right) \oplus G_{i-1} \\
H_{i} & =\mathrm{F}_{B}\left(G_{i-1}, H_{i-1}, M_{i}\right)=E\left(G_{i-1} \oplus C, H_{i-1} \mid M_{i}\right) \oplus G_{i-1} \oplus C,
\end{aligned}
$$

where $\left.{ }^{\prime}\right|^{\prime}$ represents concatenation and $c \in\{0,1\}^{n}-\left\{0^{n}\right\}$ is a constant.

A visualization of this compression function is given in Figure 4

\section{B.2 Collision Resistance of the Compression Function}

As the security proof of Hirose [13, Theorem 4] only states a collision resistance bound for a hash function built using $F^{\text {Hirose }}$, we will give a bound for the compression function itself. In particular, we will show:

Theorem 3. Let $F:=F^{\text {Hirose }}$ be a compression function as in Defintion 5 . Then,

$$
\operatorname{Adv}_{F}^{\text {COLL }}(q) \leq \frac{2 q^{2}}{\left(2^{n}-2 q\right)^{2}}+\frac{2 q}{2^{n}-2 q}
$$




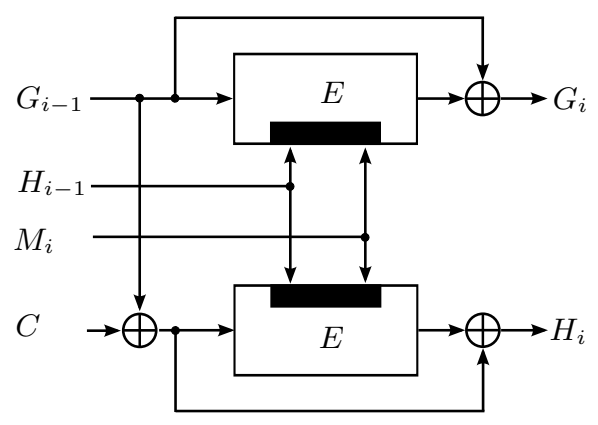

Fig. 4. The compression function $F^{\text {Hirose }}, E$ is an $(n, 2 n)$ block cipher, the small rectangle inside the cipher rectangle indicates the position of the key

In numerical terms, it means that no adversary performing less than $2^{124.55}$ oracle calls can have more than an even chance, i.e. 0.5 , in finding a collision.

Due to the special structure of the compression function, the following definition is useful for the proof.

Definition 6. A pair of distinct inputs $\left(G_{i-1}, H_{i-1}, M_{i}\right),\left(G_{i-1}^{\prime}, H_{i-1}^{\prime}, M_{i}^{\prime}\right)$ to $F^{\text {Hirose }}$ is called a matching pair if $\left(G_{i-1}^{\prime}, H_{i-1}^{\prime}, M_{i}^{\prime}\right)=\left(G_{i-1}, H_{i-1}, M_{i} \oplus C\right.$. Otherwise they are called a non-matching pair.

Note, that the proof is essentially due to Hirose [13], but as he stated it only for the hash function and not for the compression function itself. We will give a proof here for the compression function.

Proof. Let $\mathcal{A}$ be an adversary that asks $q$ queries to oracles $E, E^{-1}$. Since

$$
G_{i}=E\left(G_{i-1}, H_{i-1} \mid M_{i}\right) \oplus G_{i-1}
$$

depends both on the plaintext and the ciphertext of $E$ and one of them is fixed by a query and the other is determined by the answer, it follows that $G_{i}$ is determined randomly. We give the adversary for free the answer to the query for $H_{i}$. Let $\left(X_{i}, K_{i, 1} \mid K_{i, 2}, Y_{i}\right)$ and $\left(X_{i} \oplus C, K_{i, 1} \mid K_{i, 2}, Z_{i}\right)$ be the triplets of $E$ obtained by the $i$-th pair of queries and the corresponding answers.

For any $2 \leq i \leq q$, let $\mathrm{C}_{i}$ be the event that a colliding pair of non-matching inputs is found for $F$ with the $i$-th pair of queries. Namely, it is the event that, for some $i^{\prime}<i$

$$
F\left(X_{i}, K_{1, i}, K_{2, i}\right)=F\left(X_{i^{\prime}}, K_{1, i^{\prime}}, K_{2, i^{\prime}}\right) \text { or } F\left(X_{i^{\prime}} \oplus C, K_{1, i^{\prime}}, K_{2, i^{\prime}}\right)
$$

or

$$
F\left(X_{i} \oplus C, K_{1, i}, K_{2, i}\right)=F\left(X_{i^{\prime}}, K_{1, i^{\prime}}, K_{2, i^{\prime}}\right) \text { or } F\left(X_{i^{\prime}} \oplus C, K_{1, i^{\prime}}, K_{2, i^{\prime}}\right)
$$

which is equivalent to

$$
\begin{array}{r}
\left(Y_{i} \oplus X_{i}, Z_{i} \oplus X_{i} \oplus C\right)=\left(Y_{i^{\prime}} \oplus X_{i^{\prime}}, Z_{i^{\prime}} \oplus X_{i^{\prime}} \oplus C\right) \\
\text { or }\left(Z_{i^{\prime}} \oplus X_{i^{\prime}} \oplus C, Y_{i^{\prime}} \oplus X_{i^{\prime}}\right) .
\end{array}
$$


It follows, that

$$
\operatorname{Pr}\left[\mathrm{C}_{i}\right] \leq \frac{2(i-1)}{\left(2^{n}-(2 i-2)\right)\left(2^{n}-(2 i-1)\right)} \leq \frac{2 q}{\left(2^{n}-2 q\right)^{2}} .
$$

Let $\mathrm{C}$ be the event that a colliding pair of non-matching inputs is found for $F^{\text {Hirose }}$ with $q$ (pairs) of queries. Then,

$$
\operatorname{Pr}[\mathrm{C}] \leq \sum_{i=2}^{q} \operatorname{Pr}\left[\mathrm{C}_{i}\right] \leq \sum_{i=2}^{q} \frac{2 q}{\left(2^{n}-2 q\right)^{2}} \leq \frac{2 q^{2}}{\left(2^{n}-2 q\right)^{2}} .
$$

Now, let $\hat{\mathrm{C}}_{i}$ be the event that a colliding pair of matching inputs is found for $F$. It follows, that

$$
\operatorname{Pr}\left[\hat{\mathrm{C}}_{i}\right] \leq \frac{2}{\left(2^{n}-2 q\right)}
$$

Let $\hat{\mathrm{C}}$ be the event that a colliding pair of matching inputs is found for $F^{\text {Hirose }}$ with $q$ (pairs) of queries. Then,

$$
\operatorname{Pr}[\hat{\mathbf{C}}] \leq \sum_{i=2}^{q} \operatorname{Pr}\left[\hat{\mathbf{C}}_{i}\right] \leq \frac{2 q}{2^{n}-2 q}
$$

Since $\operatorname{Adv}_{F}^{\text {Coll }}(q)=\operatorname{Pr}[\mathrm{C} \vee \hat{\mathrm{C}}] \leq \operatorname{Pr}[\mathrm{C}]+\operatorname{Pr}[\hat{\mathrm{C}}]$, the claim follows. 